\title{
Multimode radiation from an unflanged, semi-infinite circular duct with uniform flow
}

\author{
Samuel Sinayoko, Phillip Joseph, and Alan McAlpine \\ Institute of Sound and Vibration Research, University of Southampton, Southampton SO17 1BJ, \\ United Kingdom
}

(Received 30 January 2009; revised 25 January 2010; accepted 30 January 2010)

\begin{abstract}
Multimode sound radiation from an unflanged, semi-infinite, rigid-walled circular duct with uniform subsonic mean flow everywhere is investigated theoretically. The multimode directivity depends on the amplitude and directivity function of each individual cut-on mode. The amplitude of each mode is expressed as a function of cut-on ratio for a uniform distribution of incoherent monopoles, a uniform distribution of incoherent axial dipoles, and for equal power per mode. The directivity function of each mode is obtained by applying a Lorentz transformation to the zero-flow directivity function, which is given by a Wiener-Hopf solution. This exact numerical result is compared to an analytic solution, valid in the high-frequency limit, for multimode directivity with uniform flow. The high-frequency asymptotic solution is derived assuming total transmission of power at the open end of the duct, and gives the multimode directivity function with flow in the forward arc for a general family of mode amplitude distribution functions. At high frequencies the agreement between the exact and asymptotic solutions is shown to be excellent.
\end{abstract}

(C) 2010 Acoustical Society of America. [DOI: 10.1121/1.3327814]

PACS number(s): 43.28.Py, 43.20.Mv, 43.50.Cb [AH]

Pages: 2159-2168

\section{INTRODUCTION}

\section{A. Background: Duct power estimation from limited far-field data}

Various common noise sources radiate sound into finite length ducts containing a uniform mean flow, from which the sound escapes into the far field via radiation from an unbaffled open end. Examples are exhaust mufflers, large exhaust stacks, and aircraft turbofan engines. Often one wishes to determine the sound power radiated from the duct opening, either as an index of insertion loss in order to assess silencer performance, or as a means of quantifying and ranking the total noise output for predicting community annoyance. The sound power may, in principle, be determined by integrating the normal component of sound intensity over a surface enclosing the duct exit at a large distance from the duct where the flow is quiescent. However, sometimes not all measurement locations required to perform the integration are easily accessible, as in the case of large exhaust stacks, which may be tens of meters high. In this example, the only measurements that are easy to make are close to the ground, corresponding to the rear arc or backward-radiated sound radiated at angles approaching $180^{\circ}$ to the duct axis. It is clear that a method of inferring the radiated power, at any frequency, from a small number of far-field mean square pressure measurements would be extremely useful to the noise control engineer.

\section{B. Scope of investigation}

This paper presents a theoretical and numerical study of the non-dimensional directivity function $Q(k a, \theta)$ for multimode sound radiation from the open end of an unflanged, semi-infinite, rigid-walled circular duct, in the presence of a uniform subsonic mean flow. This paper extends earlier work by Joseph and Morfey in which the effects of flow were neglected. ${ }^{1}$ The directivity function $Q(k a, \theta)$ relates the farfield mean square pressure, at any polar angle $\theta$ to the duct axis including the rear arc and at any flow Mach number, to the net sound power transmitted along the duct. The nondimensional frequency $k a$ equals $2 \pi f a / c$, where $f$ is the frequency, $a$ is the duct radius, and $c$ is the sound speed.

To calculate the modal radiation in the presence of uniform flow, a Lorentz transform is applied to the exact theoretical zero-flow modal directivity functions for sound radiation from an unflanged, semi-infinite, rigid-walled circular duct, as given by Homicz and Lordi. ${ }^{2}$ This single-mode analysis is applied in what follows to a particular family of mode amplitude distribution functions, which includes the following three special cases: (a) excitation of incident modes by incoherent monopoles uniformly distributed over a duct cross section, (b) excitation by incoherent axial dipoles uniformly distributed over a duct cross section, and (c) equal in-duct power per incident mode above cut-off. In each case, the individual modes are incoherently excited, and the contribution of evanescent modes is neglected.

This paper also presents high-frequency asymptotic expressions for $Q(k a, \theta)$ for the three source models that explicitly include the effect of uniform mean flow. Since scattering at the edge of the duct opening is neglected in the high-frequency model, this analysis is limited to the forward arc.

Simulations of $Q(\mathrm{ka}, \theta)$ as a function of $\theta$ are presented in this article, for the three incident-mode source models, at a number of representative Mach numbers (positive and negative representing exhaust and inlet conditions) in both the forward and rear arcs. Results showing $Q$ for $k a=50$ calculated from the exact result and from the high-frequency 
asymptotic result are compared with generally excellent agreement in the forward arc, not too close to the sideline directions.

This approach is used for the first time to assess the sensitivity of multimode radiation from ducts to the effects of air flow. The derivation of analytic expression for multimode far-field radiation, in which the effects of flow are included, allows decisions to be made about when flow effects should be included in numerical prediction schemes such as finite element (FE) and computational fluid dynamics (CFD) analyses. It also provides insight into the relative sensitivity of different sources (and therefore mode amplitude distributions) to the effects of flow. In particular, the analytic solutions presented here for the multimode directivity functions permit a rapid assessment of the far-field radiation angles that are most affected by flow.

\section{Previous work}

\section{Multimode radiation}

As outlined above, the problem of high-frequency radiation from ducts in which the sound field comprises many acoustic modes is common to a number of situations in which noise is a serious issue, such as the broadband noise from ducted fans and exhaust stacks. Yet almost no work has been published dealing with the characteristics and level of the noise field transmitted along the duct and its subsequent radiation to the far field. Only Rice $^{3}$ in the 1970s, and more recently Joseph and Morfey, ${ }^{1}$ explicitly addressed some of these issues for multimode sound radiation from ducts. However, both studies neglect the potentially important effect of the uniform mean flow. Rice ${ }^{3}$ derived an expression for the high-frequency mean square pressure directivity by (effectively) replacing the expression for mean square pressure involving a summation over two modal indices with a single integral over modal cut-off ratio. This simplification is based on the principle that the angles of the main radiation lobes for different modes with the same cut-off ratio are identical. Rice $^{3}$ assumed a distribution of mode amplitudes that varies as the cut-off ratio raised to some exponent. Although there is no physical justification for such a mode distribution function, the situation in which the total sound power is shared equally among the propagating modes is a special case. His analysis is restricted to the forward arc, and the effects of mean flow are neglected.

Joseph and Morfey ${ }^{1}$ presented a more general formulation for the multimode mean square pressure directivity in the absence of flow that is valid in both forward and rear arcs. The analysis was undertaken for a general family of mode distributions in which incoherent monopoles and axial dipoles uniformly distributed over the duct cross section are special cases, as is also the case of "equal energy per mode." Following a similar procedure to that adopted by Rice, ${ }^{3}$ closed-form expressions were derived for the mean square pressure directivity non-dimensionalized on the total sound power for the above source distributions that are restricted to the high-frequency limit in the forward arc.

The present paper extends the work of Joseph and Morfey ${ }^{1}$ to include the effects of a uniform mean flow that is everywhere the same. Flow effects are shown to substantially distort the directivity for some mode amplitude distributions. The analysis shows that, despite the amplification effects on the sound power (convective amplification) and the distortion of the directivity function by the mean flow, the mean square pressure normalized on sound power in the highfrequency limit can be still expressed in closed form.

\section{Single-mode radiation}

There is far more published work associated with singlemode radiation from ducts (compared to work reported on multimode radiation). In the absence of mean flow, Homicz and Lordi $^{2}$ gave the exact modal directivity functions for sound radiation from an unflanged, semi-infinite, rigidwalled circular duct. These are derived using the WienerHopf technique. Homicz and Lordi ${ }^{2}$ outlined how the corresponding directivity functions with a uniform flow everywhere can be determined from the no flow solutions. Their approach is utilized in this current work (see Sec. II A).

We note here that other Wiener-Hopf solutions of sound radiation from flow ducts have been reported since the work by Homicz and Lordi. ${ }^{2}$ In Ref. 4 the far-field sound radiation from a semi-infinite duct is derived for the case when the (uniform) flow velocities inside and outside the duct are different. Munt ${ }^{4}$ modeled the separation between the two fluids by a vortex sheet. In Ref. 4 a comparison between experimental measurements and predictions of the far-field pressure showed excellent agreement. He later extended his result to give the near-field solution. ${ }^{5}$ Rienstra ${ }^{6}$ examined sound radiation from a semi-infinite annular duct immersed in a uniform flow, where the centerbody extends downstream from the open end of the duct. More recently these solutions have been extended by Gabard and Astley, ${ }^{7}$ who derived the sound radiated from a semi-infinite annular duct for the case when the (uniform) flow velocities inside and outside the duct are different. In addition to providing the exact solution for the single-mode directivity, Gabard and Astley ${ }^{7}$ also gave an example of multimode directivity for equal power per mode. However, they did not derive any simple analytical expressions for the far-field multimode directivity with flow, which is the objective of the work reported in this paper.

\section{Summary of simplifying assumptions used in the modeling of the multimode radiation from ducts with uniform flow}

In this work, a number of important simplifications are made in the analysis of the multimode sound radiation from ducts with uniform flow. The main simplifying assumptions are listed as follows.

(1) The duct is assumed to be straight, circular, and of semiinfinite length.

(2) The duct walls are rigid and have zero thickness.

(3) The duct is assumed to be free of absorbing liners and other forms of dissipation.

(4) The mean flow is assumed to be steady, inviscid, and isentropic. The mean flow is assumed to be uniform ev- 


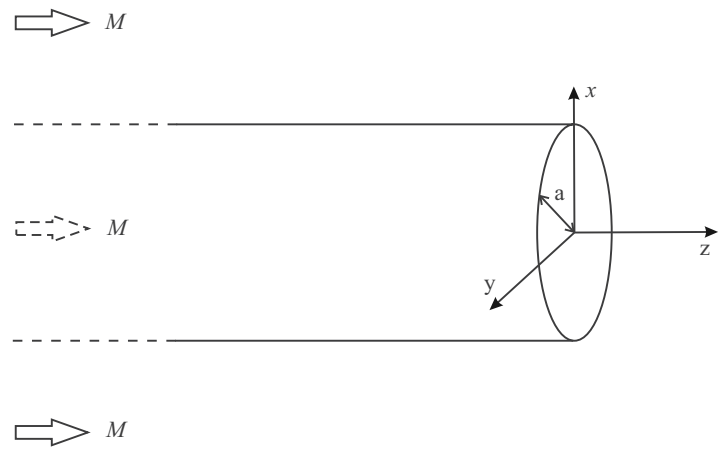

FIG. 1. Circular duct of radius $a$ immersed in a uniform flow of Mach number $M$.

erywhere so that no shear layers exist downstream of the duct, and there are no boundary layers at the duct walls.

A sketch of the semi-infinite, circular duct of radius $a$ immersed in a uniform mean flow of Mach number $M$ is shown in Fig. 1. As a consequence of (1), axial standing waves can be neglected. Assumption (2) allows the effects of diffraction and scattering to be neglected in the high- $k a$ analytic radiation models developed in this paper. We emphasize, however, that the full effects of diffraction at the duct lip are included in the general model formulation to allow the prediction of rear arc radiation. As a consequence of (3) and (4) energy is conserved, so that in-duct sound power equals the sound power radiated to the far field. In the absence of any jet shear layers (since the flow is everywhere uniform), energy dissipation mechanisms identified by, for example, Bechert, ${ }^{8}$ in which acoustic energy is dissipated by the vorticity in the jet shear layers, are not included. Also as a consequence of (4), complications in the prediction of farfield radiation arising from shear layer refraction, and scattering owing to unsteady, turbulent flow in jet shear layers, therefore, do not arise. Thus, in this work, the consequences of including the mean flow are limited to convection effects.

\section{FAR-FIELD PRESSURE DIRECTIVITY WITH FLOW}

Consider an unflanged, semi-infinite, rigid-walled circular duct of radius $a$, immersed in a uniform flow of Mach number $M$, which is assumed to be identical inside and outside the duct. Inside the duct, the position is specified in terms of cylindrical polar coordinates $(r, \phi, z)$, as sketched in Fig. 2. Outside the duct, the position is specified in terms of

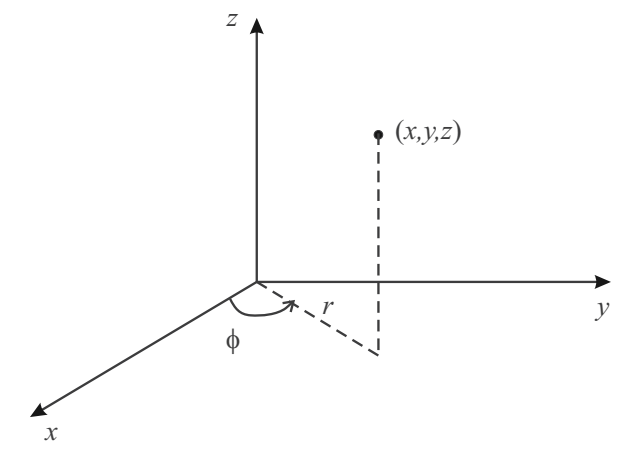

FIG. 2. Cylindrical polar coordinate system $(r, \phi, z)$.

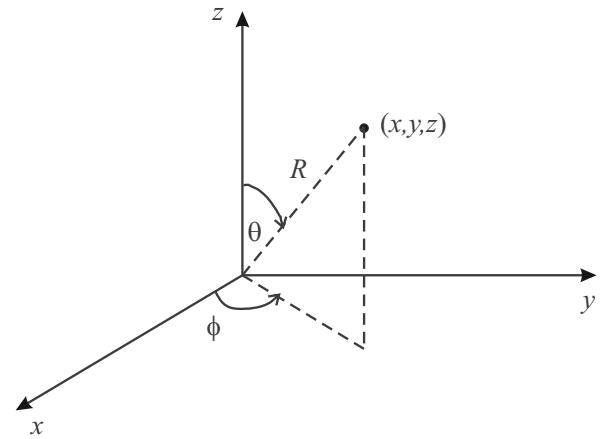

FIG. 3. Spherical polar coordinate system $(R, \theta, \phi)$.

spherical polar coordinates $(R, \theta, \phi)$, as sketched in Fig. 3 .

At a single frequency $\omega$, the acoustic pressure field propagating toward the open end of a semi-infinite duct containing a uniform mean flow may be expressed in the form

$$
p(r, \phi, z, t)=\sum_{(m, n) \in \mathcal{O}} A_{m n} \Psi_{m n}(r, \phi) e^{j\left(\omega t-k_{z, m n} z\right)},
$$

where the mode order $(m, n)$ is restricted to the set of cut-on modes, denoted by $\mathcal{O}$. In Eq. (1) $A_{m n}$ is the modal pressure amplitude at the source plane (say, $z=0$ ), $\Psi_{m n}$ is the orthonormal mode shape function of the duct defined such that

$$
\begin{aligned}
& \frac{1}{S} \int_{0}^{2 \pi} \int_{0}^{a} \Psi_{m n}(r, \phi) \Psi_{m^{\prime} n^{\prime}}^{*}(r, \phi) r \mathrm{~d} r \mathrm{~d} \phi \\
&=\left\{\begin{array}{ll}
0 & (m, n) \neq\left(m^{\prime}, n^{\prime}\right) \\
1 & (m, n)=\left(m^{\prime}, n^{\prime}\right)
\end{array}\right\},
\end{aligned}
$$

where $S=\pi a^{2}$ is the cross-sectional area of the duct, and $k_{z, m n}$ denotes the axial wavenumber of mode $(m, n)$ given by

$$
k_{z, m n}=\frac{\left(\alpha_{m n}-M\right) k}{\beta^{2}} \text { where } \alpha_{m n}=\sqrt{1-\left(\frac{\kappa_{m n}}{k}\right)^{2} \beta^{2}} .
$$

In Eq. (3), $k=\omega / c$ denotes the free field acoustic wavenumber, $\beta=\sqrt{1-M^{2}}, \alpha_{m n}$ is the modal cut-on ratio, and $\kappa_{m n}=j_{m n} / a$ is the mode transverse wavenumber where $j_{m n}$ equals the value of the $n$th turning point of the Bessel function of the first kind of order $m$. The modal cut-on ratio $\alpha_{m n}$ is in the range $0 \leq \alpha_{m n} \leq 1$ for propagating modes, where $\alpha_{m n}=0$ is the cut-on/cut-off transition point. ${ }^{9}$ For evanescent modes, $\alpha_{m n}$ is purely imaginary, and the modes transport no sound power to the far field. Therefore, in this paper, evanescent modes are neglected, and in Eq. (1) the sum is restricted to the set of cut-on modes.

Also at a single frequency $\omega$, we define an in-duct to far-field non-dimensional transfer function $H_{m n}(k a, \theta)$, such that the far-field pressure may be written as

$$
p_{f}(R, \theta, \phi, t)=\frac{a}{R} \sum_{(m, n) \in \mathcal{O}} A_{m n} H_{m n}(k a, \theta) e^{j(\omega t-m \phi-k R)} .
$$

We now assume that the excitation is broadband in origin such that the modal amplitudes are random variables with respect to time $t$. This is a realistic assumption in the case of, for example, excitation by the incoherent sources distributed over the rotor blades and stator vanes in a turbofan engine 
arising from interaction with turbulent flow. In practice, a single realization of the modal amplitudes $A_{m n}$ may be obtained by taking the Fourier transform of the pressure at the source plane over a short time interval. For broadband excitation, the modes are assumed to be incoherent; i.e.,

$$
E\left\{A_{m n} A_{m^{\prime} n^{\prime}}^{*}\right\}=0 \quad \text { if } \quad(m, n) \neq\left(m^{\prime}, n^{\prime}\right)
$$

in which case the mean square pressure becomes

$$
\overline{p_{f}^{2}}(R, \theta)=\frac{1}{2}\left(\frac{a}{R}\right)^{2} \sum_{(m, n) \in \mathcal{O}}\left|H_{m n}(k a, \theta)\right|^{2} E\left\{\left|A_{m n}\right|^{2}\right\},
$$

where for broadband noise $\overline{p_{f}^{2}}$ is the far-field mean square pressure in a small specified frequency bandwidth, and similarly $E\left\{\left|A_{m n}\right|^{2}\right\}$ is the expectation of the modulus squared of the modal amplitudes in the same frequency bandwidth. Assuming incoherent modes Eq. (5) simplifies the expression for the far-field mean square pressure $\overline{p_{f}^{2}}$, and ensures that the radiated field is axisymmetric; i.e., there is no dependence on azimuthal angle $\phi$.

\section{A. Transfer function for modal radiation from ducts with flow}

In this section, the modal radiation transfer function $H_{m n}$ for ducts immersed in a uniform flow is related to that for modal radiation in the absence of flow, denoted by $H_{m n}^{0}$. Homicz and Lordi $^{2}$ gave the far-field expression for $H_{m n}^{0}$, which is derived using the Wiener-Hopf technique. We note that an approximate zero-flow transfer function may also be obtained using the Rayleigh integral, whose validity is restricted to the forward arc, which has been formulated previously, for example, by Tyler and Sofrin. ${ }^{10}$

Following the procedure in Ref. $2, H_{m n}$ is obtained by Lorentz transformation of the zero-flow transfer function $H_{m n}^{0}$. This type of approach, previously used by Homicz and Lordi, ${ }^{2}$ has been recently examined in more detail by Chapman. ${ }^{11}$ In essence, $M>0$ represents an exhaust problem, where sound is radiated in the direction of the flow, whereas $M<0$ represents an inlet problem, where sound is radiated against the oncoming flow.

In the case of $M>0$, following the procedure outlined in Ref. 2, in our notation we obtain

$$
H_{m n}(k a, \theta)=\frac{\beta}{\sqrt{1-M^{2} \sin ^{2} \theta}} H_{m n}^{0}\left(k a / \beta, \tan ^{-1}(\beta \tan \theta)\right),
$$

where $H_{m n}^{0}$ is the far-field transfer function of mode $(m, n)$ in the absence of flow $(M=0)$.

In the case of $M<0$, the singularity in pressure on the leading edge of the open end of the duct requires that the Lorentz transformation is applied instead to the zero-flow velocity potential solution. The pressure solution is then obtained from the velocity potential via the acoustic momentum equation. Again following the procedure in Ref. 2, in our notation we obtain
TABLE I. Details of source models in a circular duct with uniform flow. Note that $P_{\mu \nu \gamma}^{2}$ has dimensions of pressure squared in a small specified frequency bandwidth. In this small frequency bandwidth, $\overline{Q_{s}^{2}}$ is the mean square volume velocity per unit area due to the monopole distribution, $\overline{F_{s}^{2}}$ is the mean square force per unit area due to the dipole distribution, and in the case of equal power per mode, $W_{0}$ is the power transmitted by each mode.

\begin{tabular}{lccc}
\hline \hline & Monopole & Dipole & Equal power per mode \\
\hline$(\mu, \nu, \gamma)$ & $(0,1,2)$ & $(1,0,2)$ & $(0,1,1)$ \\
$P_{\mu \nu \gamma}^{2}$ & $\left(\rho_{0} c_{0}\right)^{2} \overline{Q_{s}^{2}} / 2$ & $\overline{F_{s}^{2}} / 2$ & $2 \rho_{0} c_{0} W_{0} / S$ \\
\hline \hline
\end{tabular}

$$
\begin{aligned}
H_{m n}(k a, \theta)= & \frac{\beta}{1-M \alpha_{m n}} \frac{\sqrt{1-M^{2} \sin ^{2} \theta}-M \cos \theta}{1-M^{2} \sin ^{2} \theta} \\
& \times H_{m n}^{0}\left(k a / \beta, \tan ^{-1}(\beta \tan \theta)\right),
\end{aligned}
$$

where it is noted that this equation differs slightly from the equation given by Homicz and Lordi, most likely due to a typographical error in Ref. 2. Our result is recovered by replacing $\cos \psi$ in the expression in Ref. 2 with $\cos \tilde{\psi}$.

\section{B. Modal amplitude distributions}

In this paper the behavior of the far-field mean square pressure $\overline{p_{f}^{2}}$ is investigated for a particular family of mode distribution functions $E\left\{\left|A_{m n}\right|^{2}\right\}$. The mode amplitude distribution function may be obtained by generalizing the approach taken by Joseph et al. ${ }^{12}$ The result is

$$
E\left\{\left|A_{m n}\right|^{2}\right\}=P_{\mu \nu \gamma}^{2}\left(\frac{M-\alpha_{m n}}{1-M^{2}}\right)^{2 \mu}\left(\frac{1-\alpha_{m n} M}{1-M^{2}}\right)^{2 \nu} \frac{1}{\alpha_{m n}^{\gamma}},
$$

where $P_{\mu \nu \gamma}$ is a measure of source strength, and $(\mu, \nu, \gamma)$ denotes a trio of indices that define the source model. Written in full, $E\left\{\left|A_{m n}\right|^{2}\right\}$ is listed below in Eq. (10) for the following special cases: (a) a uniform distribution of incoherent monopoles $(\mu, \nu, \gamma)=(0,1,2)$, (b) a uniform distribution of incoherent axial dipoles $(\mu, \nu, \gamma)=(1,0,2)$, and (c) equal power per mode $(\mu, \nu, \gamma)=(0,1,1)$.

(a) $E\left\{\left|A_{m n}\right|^{2}\right\}=P_{012}^{2}\left(\frac{1-\alpha_{m n} M}{1-M^{2}}\right)^{2} \frac{1}{\alpha_{m n}^{2}}$,

(b) $E\left\{\left|A_{m n}\right|^{2}\right\}=P_{102}^{2}\left(\frac{M-\alpha_{m n}}{1-M^{2}}\right)^{2} \frac{1}{\alpha_{m n}^{2}}$,

(c) $E\left\{\left|A_{m n}\right|^{2}\right\}=P_{011}^{2}\left(\frac{1-\alpha_{m n} M}{1-M^{2}}\right)^{2} \frac{1}{\alpha_{m n}}$.

The source strength term and indices $(\mu, \nu, \gamma)$ for each mode amplitude distribution are summarized in Table I.

Equation (9) is more general than the source models derived previously by Joseph et al., ${ }^{12}$ but at the expense of having to include an additional exponent, $\gamma$, which allows for the equal power per mode model to be included in the general family of mode amplitude distribution functions.

Sinayoko et al.: Multimode radiation from ducts with flow 


\section{MULTIMODE DIRECTIVITY FACTOR $Q(k a, \theta)$ WITH FLOW}

As in Ref. 1 the non-dimensional multimode directivity function is defined by

$$
Q(k a, \theta)=\frac{4 \pi R^{2}}{\rho_{0} c_{0} E\left\{W_{f}\right\}} \overline{p_{f}^{2}}(R, \theta),
$$

where $E\left\{W_{f}\right\}$ denotes the expected value of the radiated acoustic power. Following the definition of generalized acoustic intensity given by, for example, Morfey, ${ }^{13}$ it can be shown that the radiated power is given by

$$
E\left\{W_{f}\right\}=\frac{4 \pi R^{2}}{\rho_{0} c_{0}} \int_{0}^{\pi} \overline{p_{f}^{2}}(R, \theta) F(\theta) \sin \theta \mathrm{d} \theta,
$$

where, after considerable algebra, $F(\theta)$ is given by

$$
F(\theta)=\frac{\beta^{4} \sqrt{1-M^{2} \sin ^{2} \theta}}{2\left(\sqrt{1-M^{2} \sin ^{2} \theta}-M \cos \theta\right)^{2}} .
$$

Note that for zero-flow, $F(\theta)=1 / 2$, and Eq. (12) reduces to the classical result for radiated sound power in a medium at rest. The function $F$ incorporates two effects on the intensity by the mean flow. One is that it resolves the intensity in the radial direction, since in the presence of flow the intensity vector is no longer radially orientated. The second is that it includes the effects of convective amplification in which the sound intensity is modified by the presence of flow.

Combining Eqs. (11) and (12) shows that $Q$ satisfies the normalization condition

$$
\int_{0}^{\pi} Q(k a, \theta) F(\theta) \sin \theta \mathrm{d} \theta=1 .
$$

Evaluation of Eq. (11) using Eqs. (6)-(8) and (10) is a computationally intensive procedure for large $k a$ and $M$. For example, at $k a=50$, there are approximately 650 cut-on modes at $M=0$. This number increases as $\left(1-M^{2}\right)^{-1 / 2}$ as $M$ increases. Thus, there is a need for a simple analytic formula that provides a good approximation to $Q(k a, \theta)$ in the highfrequency limit, for the family of sound sources described in Sec. II B.

\section{ANALYTIC EXPRESSION FOR $Q(k a, \theta)$ IN THE HIGH-ka LIMIT}

In this section, analytic expressions are derived for the non-dimensional multimode directivity function $Q$ (11) in the presence of flow for the general family of source distributions given in Sec. II B.

In order to determine the radiated sound power $E\left\{W_{f}\right\}$ for use in the evaluation of $Q$, we make the assumption that the incident transmitted sound power in the duct and that radiated from the open end are equal. Thus, we first consider the sound power $E\left\{W_{m n}\right\}$ transmitted by a single-mode wave traveling along the duct with flow. Following Morfey, ${ }^{13}$

$$
E\left\{W_{m n}\right\}=\frac{S}{2 \rho_{0} c_{0}} \frac{\beta^{4} \alpha_{m n}}{\left(1-\alpha_{m n} M\right)^{2}} E\left\{\left|A_{m n}\left(\alpha_{m n}\right)\right|^{2}\right\},
$$

where $E\left\{\left|A_{m n}\right|^{2}\right\}$ is a function of $\alpha_{m n}$ given by Eq. (9) and is defined by the indices $(\mu, \nu, \gamma)$.

\section{A. In-duct sound power transmission between $\theta$ and $\boldsymbol{\theta}+\boldsymbol{\delta} \boldsymbol{\theta}$}

Rice et al. ${ }^{14}$ examined how duct modes' propagation angles are related to sound radiation. They derived the angle of propagation with respect to the duct axis of the wavefronts connected to modes in a circular duct with uniform mean flow. In this paper the modal propagation angle $\theta$ is equivalent to what Rice et al. ${ }^{14}$ referred to as the "resultant axial propagation angle in the duct." This angle is not normal to the wavefronts owing to the convective effect of the mean flow.

The in-duct sound power transmitted along the duct by modes between propagation angles $\theta$ and $\theta+\delta \theta$ is the sum of the modal powers transmitted by each mode whose propagation angle $\theta_{m n}$ lies between $\theta$ and $\theta+\delta \theta$; i.e.,

$$
\delta W(\theta)=\sum_{(m, n) \in \mathcal{O}_{\theta}} W_{m n},
$$

where

$$
\mathcal{O}_{\theta}=\left\{(m, n) \in \mathcal{O} \mid \theta \leq \theta_{m n} \leq \theta+\delta \theta\right\} .
$$

Combining Eqs. (15) and (16) gives

$$
E\{\delta W(\theta)\}=\frac{S}{2 \rho_{0} c_{0}} \sum_{(m, n) \in \mathcal{O}_{\theta}} \frac{\beta^{4} \alpha_{m n}}{\left(1-\alpha_{m n} M\right)^{2}} E\left\{\left|A_{m n}\left(\alpha_{m n}\right)\right|^{2}\right\} .
$$

The propagation angle $\theta_{m n}$ can be related to $\alpha_{m n}$ (and $M$ ) using the following result from Ref. 14:

$$
\cos \theta_{m n}=\frac{\alpha_{m n} \beta}{\sqrt{1-M^{2} \alpha_{m n}^{2}}} .
$$

For each mode $(m, n) \in \mathcal{O}_{\theta}$, since $\delta \theta \ll 1$, we have $\theta_{m n} \approx \theta$, and Eq. (19) may be rearranged giving

$$
\alpha(\theta) \approx \frac{\cos \theta}{\sqrt{1-M^{2} \sin ^{2} \theta}} .
$$

Substituting Eq. (20) into Eq. (18) gives

$$
E\{\delta W(\theta)\}=\frac{S}{2 \rho_{0} c_{0}} \frac{\beta^{4} \alpha(\theta)}{(1-\alpha(\theta) M)^{2}} E\left\{\left|A_{m n}(\alpha(\theta))\right|^{2}\right\} \delta N(\theta),
$$

where $\delta N(\theta)$ denotes the number of modes with propagation angle between $\theta$ and $\theta+\delta \theta$, such that $\delta N / N$ is small, where $N$ is the total number of propagating modes.

We now take the limit as $k a \rightarrow \infty$ to obtain an asymptotic expression for $\delta N$ to enable Eq. (21) to be evaluated analytically. The derivation of $\delta N(\theta)$ is presented in the Appendix. It is shown that as $k a \rightarrow \infty$, 


$$
\delta N(\theta)=\left(\frac{1}{2} k a\right)^{2} \frac{\sin 2 \theta}{\left(1-M^{2} \sin ^{2} \theta\right)^{2}} \delta \theta .
$$

Substituting this asymptotic expression for $\delta N(\theta)$ in Eq. (21) leads to the following analytic expression for the in-duct sound power transmitted between the range of angles $\theta$ and $\theta+\delta \theta$ :

$$
\begin{aligned}
E\{\delta W(\theta)\}= & \frac{S}{2 \rho_{0} c_{0}}\left(\frac{1}{2} k a\right)^{2} \frac{\sin 2 \theta}{\left(1-M^{2} \sin ^{2} \theta\right)^{2}} \\
& \times \frac{\beta^{4} \alpha(\theta)}{(1-\alpha(\theta) M)^{2}} E\left\{\left|A_{m n}(\alpha(\theta))\right|^{2}\right\} \delta \theta,
\end{aligned}
$$

which is valid in the high- $k a$ limit. Thus the total transmitted power, in the limit as $k a \rightarrow \infty$, can be evaluated by an integral over polar angle $\theta$ instead of the modal summation in Eq. (18).

\section{B. Sound power radiation between polar angles $\theta$ and $\theta+\delta \theta$}

Following Eq. (12), the expected value for the far-field sound power $E\left\{\delta W_{f}(\theta)\right\}$ radiated between angles $\theta$ and $\theta$ $+\delta \theta$ is given by

$$
E\left\{\delta W_{f}(\theta)\right\}=\frac{4 \pi R^{2}}{\rho_{0} c_{0}} \overline{p_{f}^{2}}(R, \theta) F(\theta) \sin \theta \delta \theta .
$$

\section{Relation between far-field power and in-duct power}

For the case where the flow is uniform everywhere, and assuming that no energy is lost at the open end of the duct or back-reflected, the power radiated between the range of angles $\theta$ and $\theta+\delta \theta$ is equal to the power transported between this range of angles inside the duct; i.e.,

$$
\delta W_{f}(\theta)=\delta W(\theta) .
$$

This is a good approximation for multimode sound propagation at high $k a$ since only comparatively few modes are near cut-off and hence are significantly reflected back into the duct. ${ }^{13}$ These modes radiate most strongly at angles close to $90^{\circ}$, and therefore the analysis is expected to be least accurate for radiation angles close to $90^{\circ}$ to the duct axis.

\section{Multimode far-field directivity in the high-ka limit}

Equating the expression for in-duct sound power (23) to the expression for radiated sound power (24), and using Eqs. (13) and (20) for $F$ and $\alpha$, give an explicit expression for the mean square pressure as

$$
\begin{aligned}
& \overline{p_{f}^{2}}(R, \theta) \\
& \quad=\frac{1}{2}\left(\frac{a}{R}\right)^{2}\left(\frac{1}{2} k a\right)^{2} \frac{\cos ^{2} \theta}{\left(1-M^{2} \sin ^{2} \theta\right)^{2}} E\left\{\left|A_{m n}(\alpha(\theta))\right|^{2}\right\} .
\end{aligned}
$$

Noting Eqs. (10a)-(10c) for $E\left\{\left|A_{m n}\right|^{2}\right\}$ the mean square pressures for the three source distributions are given by

$$
\text { (a) } \begin{aligned}
\overline{p_{f}^{2}}(R, \theta)= & \frac{1}{2}\left(\frac{a}{R}\right)^{2}\left(\rho_{0} c_{0}\right)^{2} \frac{\overline{Q_{s}^{2}}}{2}\left(\frac{1}{2} k a / \beta\right)^{2} \\
& \times \frac{\left(\sqrt{1-M^{2} \sin ^{2} \theta}-M \cos \theta\right)^{2}}{\beta^{2}\left(1-M^{2} \sin ^{2} \theta\right)^{2}},
\end{aligned}
$$

(b) $\overline{p_{f}^{2}}(R, \theta)=\frac{1}{2}\left(\frac{a}{R}\right)^{2} \frac{\overline{F_{s}^{2}}}{2}\left(\frac{1}{2} k a / \beta\right)^{2}$

$$
\times \frac{\left(M \sqrt{1-M^{2} \sin ^{2} \theta}-\cos \theta\right)^{2}}{\beta^{2}\left(1-M^{2} \sin ^{2} \theta\right)^{2}},
$$

$$
\text { (c) } \begin{aligned}
\overline{p_{f}^{2}}(R, \theta)= & \frac{1}{2}\left(\frac{a}{R}\right)^{2}\left(\frac{2 \rho_{0} c_{0} W_{0}}{S}\right)\left(\frac{1}{2} k a / \beta\right)^{2} \\
& \times \frac{\left(\sqrt{1-M^{2} \sin ^{2} \theta}-M \cos \theta\right)^{2} \cos \theta}{\beta^{2}\left(1-M^{2} \sin ^{2} \theta\right)^{5 / 2}} .
\end{aligned}
$$

\section{E. Multimode sound power in the high-ka limit}

Now utilizing the expression for generalized directivity given by Eq. (26), the radiated sound power defined by Eq. (12) is now evaluated. Equating the radiated sound power to the in-duct transmitted power, the expected value of the source power is obtained by integrating the elementary sound power contributions $E\{\delta W(\theta)\}$ given by Eq. (23) between $\theta=0$ and $\pi / 2$. Thus, as $k a \rightarrow \infty$, contributions to the sound power from angles between $\pi / 2$ and $\pi$ are neglected (rear arc), and the expected value of the source power is given by

$$
\begin{aligned}
E\{W\}= & \frac{S}{2 \rho_{0} c_{0}}\left(\frac{k a}{2}\right)^{2} \int_{0}^{\pi / 2} \frac{\sin 2 \theta}{\left(1-M^{2} \sin ^{2} \theta\right)^{2}} \\
& \times \frac{\beta^{4} \alpha(\theta)}{(1-\alpha(\theta) M)^{2}} E\left\{\left|A_{m n}(\alpha(\theta))\right|^{2}\right\} \mathrm{d} \theta .
\end{aligned}
$$

Substituting Eqs. (10a)-(10c) for $E\left\{\left|A_{m n}\right|^{2}\right\}$ the integration over $\theta$ can be determined analytically for the three source distributions, which gives the following expressions for the sound power in the high- $k a$ limit:

$$
\begin{aligned}
& \text { (a) } E\{W\}=\frac{\rho_{0} c_{0} S \overline{Q_{s}^{2}}}{2}\left(\frac{1}{2} k a / \beta\right)^{2} \text {, } \\
& \text { (b) } E\{W\}=\frac{S \overline{F_{s}^{2}}}{2 \rho_{0} c_{0}}\left(\frac{1}{2} k a / \beta\right)^{2} \\
& \times \frac{2 \beta^{2} \ln (1-M)+2 M+M^{2}-M^{3}-M^{4}}{M^{3}},
\end{aligned}
$$

$$
\text { (c) } E\{W\}=W_{0}\left(\frac{1}{2} k a / \beta\right)^{2} \text {. }
$$

Noting from Rice ${ }^{15}$ that $N=\left(\frac{1}{2} k a / \beta\right)^{2}$ is the high- $k a$ asymptotic expression for the total number of propagating modes in the duct, the remaining factors represent the average sound power per mode.

Sinayoko et al:: Multimode radiation from ducts with flow 
Equation (29) can be used to compute the convective amplification factor $E\{W\} / E\left\{\left.W\right|_{M=0}\right\}$, which is plotted in Fig. 4 versus Mach number $M$ for the three source models under investigation. The figure shows that the amplification factor is identical for the source models having a uniform distribution of incoherent monopoles and equal power per mode. In these cases, the amplification factor is symmetrical with respect to $M=0$, and the sound power increases identically with Mach number irrespective of the flow direction. How- ever, the amplification factor for the uniform distribution of incoherent axial dipoles is not symmetric about $M=0$, and reaches a minimum value of about 0.7 at $M=0.4$.

\section{F. Non-dimensional directivity factor $Q$ in the high-ka limit}

Substituting the analytical expressions of Eqs. (26) and (28) into the definition of $Q(k a, \theta)$ given by Eq. (11) leads to

$$
Q(k a, \theta)=\frac{4 E\left\{\left|A_{m n}(\alpha(\theta))\right|^{2}\right\} \cos ^{2} \theta /\left(1-M^{2} \sin ^{2} \theta\right)^{2}}{\beta^{4} \int_{0}^{\pi / 2} \alpha(\theta) E\left\{\left|A_{m n}(\alpha(\theta))\right|^{2}\right\} \sin 2 \theta /\left[\left(1-M^{2} \cos ^{2} \theta\right)^{2}(1-\alpha(\theta) M)^{2}\right] \mathrm{d} \theta} .
$$

In the high-frequency limit $k a \rightarrow \infty$, the expression for $Q(k a, \theta)$ can be evaluated analytically for each of the three source models under investigation, i.e., for the following special cases: (a) a uniform distribution of incoherent monopoles, (b) a uniform distribution of incoherent axial dipoles, and (c) equal power per mode. The results are

$$
\begin{aligned}
& \text { (a) } Q=2 \frac{\left(\sqrt{1-M^{2} \sin ^{2} \theta}-M \cos \theta\right)^{2}}{\beta^{2}\left(1-M^{2} \sin ^{2} \theta\right)^{2}} \text {, } \\
& \text { (b) } Q=\frac{2}{\beta^{2}} \frac{M^{3}}{2 M+M^{2}-M^{3}-M^{4}+2 \beta^{2} \ln (1-M)} \\
& \times \frac{\left(M \sqrt{1-M^{2} \sin ^{2} \theta}-\cos \theta\right)^{2}}{\left(1-M^{2} \sin ^{2} \theta\right)^{2}}, \\
& \text { (c) } Q=4 \frac{\left(\sqrt{1-M^{2} \sin ^{2} \theta}-M \cos \theta\right)^{2}}{\beta^{2}\left(1-M^{2} \sin ^{2} \theta\right)^{5 / 2}} \cos \theta \text {. }
\end{aligned}
$$

Note that these high- $k a$ results are valid for all subsonic Mach numbers $-1<M<1$, i.e., independent of flow direction.

At low Mach numbers, to leading order in $M$, the expressions for $Q$ are approximately

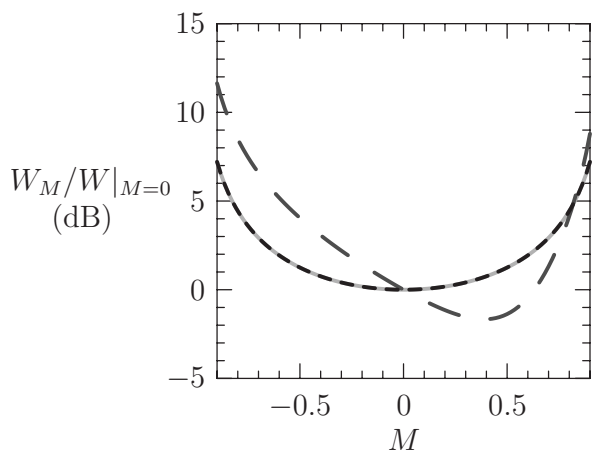

FIG. 4. In-duct power with uniform flow normalized by the power in the absence of flow versus Mach number. Results are shown for the source models: (a) a uniform distribution of incoherent monopoles (solid line), (b) a uniform distribution of incoherent axial dipoles (long-dashed line), and (c) equal power per mode (short-dashed line).
(a) $Q \sim 2-4 M \cos \theta$,
(b) $Q \sim 6 \cos ^{2} \theta+3 M\left(3 \cos ^{2} \theta-4 \cos \theta\right)$,
(c) $Q \sim 4 \cos \theta-8 M \cos \theta$.

Equations (32a)-(32c) suggest that the effect of flow on the directivity functions is most pronounced on axis, $\theta=0^{\circ}$, in the direction of flow. Note that as $M \rightarrow 0$,
(a) $Q \rightarrow 2$,
(b) $Q \rightarrow 6 \cos ^{2} \theta$,
(c) $Q \rightarrow 4 \cos \theta$,

which are the directivity factors derived previously by Joseph and Morfey ${ }^{1}$ for zero-flow.

\section{RESULTS}

The directivity function $Q(k a, \theta)$ is plotted in Figs. 5-7 versus polar angle $\theta$ at $k a=50$, for a uniform distribution of incoherent monopoles, a uniform distribution of incoherent axial dipoles, and equal power per mode, respectively. In each figure are plotted the results at Mach numbers of $M=$ $-0.9,-0.3,0,0.3$, and 0.9 . In each polar plot the solid line shows the exact directivity function and the dashed line shows the high-frequency asymptotic solutions given by Eqs. (31a)-(31c), which are valid in the forward arc. Note that for the exact results the modal radiation transfer function $H_{m n}$ used here has been computed from the transfer function in the absence of flow $H_{m n}^{0}$, given by Homicz and Lordi, ${ }^{2}$ using either Eq. (7) or Eq. (8) depending on the sign of $M$, whereas the asymptotic solutions of Eqs. (31a)-(31c) are valid for all $M$. The agreement between the exact and high-frequency asymptotic solution is excellent up to about $80^{\circ}$.

For a uniform distribution of incoherent monopoles, the directivity is omnidirectional in the forward arc for $M=0$ [Fig. 5(c)]. For $M<0$, as the flow speed increases, $Q$ increases approximately uniformly over the forward arc [Figs. 5(a) and 5(b)]. Different behavior is observed for $M>0$. In this case, as the flow speed increases, $Q$ decreases at polar angles close to the duct axis and increases at angles further away from the duct axis [Figs. 5(d) and 5(e)]. 

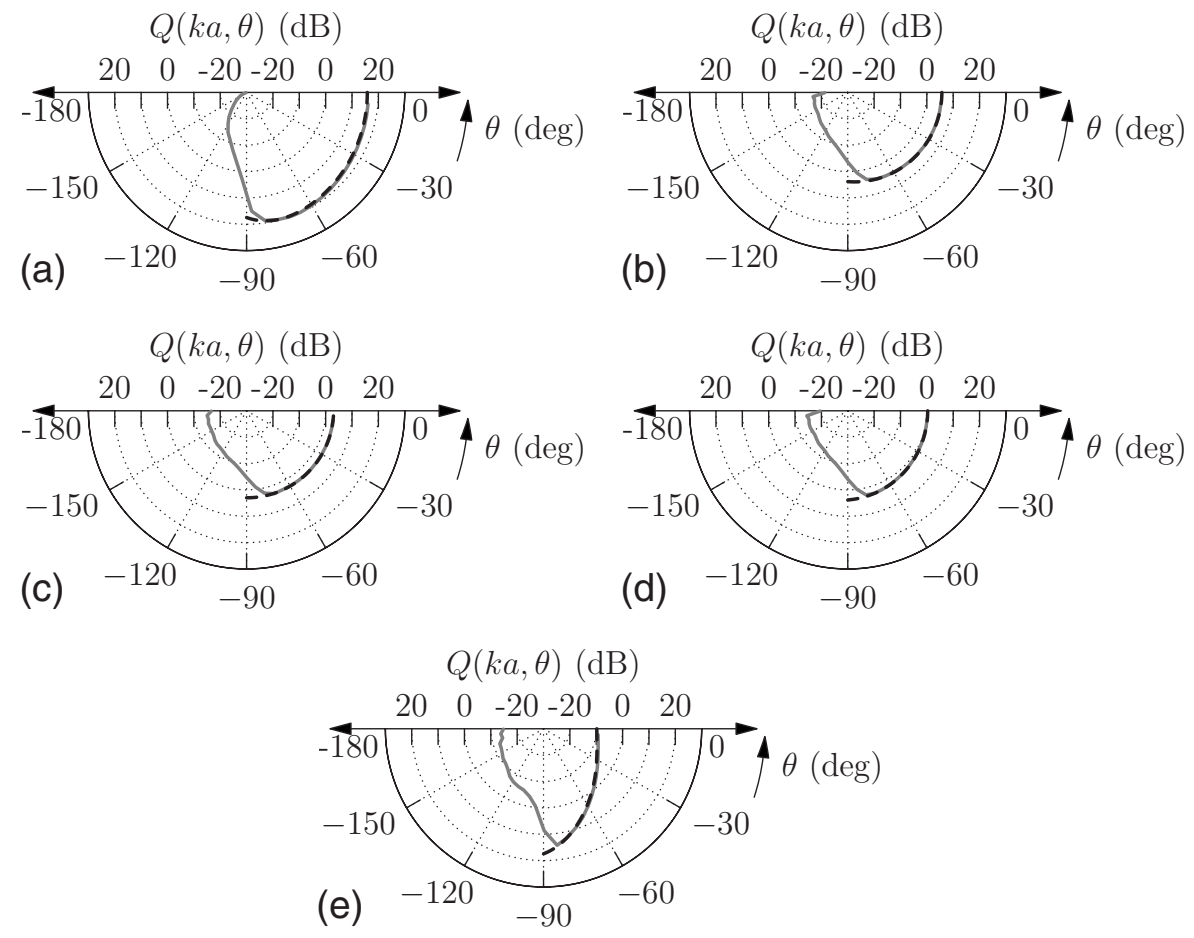

FIG. 5. [(a)-(e)] Multimode directivity function $Q(k a, \theta)$ for sound radiated from a circular duct with uniform flow. The source is a uniform distribution of incoherent monopoles. Results are shown for $k a=50$ and for five different values of Mach number $M$. The solid line represents the exact numerical solution given by Eq. (11) (see Sec. III). The dashed line represents the high-frequency asymptotic solution given by Eq. (31a).

For a uniform distribution of incoherent axial dipoles, the radiation pattern exhibits small deviations from omnidirectional behavior over the forward arc for $M=0$ [Fig. 6(c)]. However, for $M<0$, as the flow speed increases, the directivity pattern tends to become more uniform in the forward arc [Figs. 6(a) and 6(b)]. By contrast, for $M>0$, the directivity drops to zero at certain angles, and radiates more effi- ciently away from the duct axis as the flow speed increases [Figs. 6(d) and 6(e)]. From Eq. (31b), the angular position of the nulls in the directivity pattern is given by

$$
\cos \theta_{0}=\frac{M}{\sqrt{1-M^{2}}}
$$
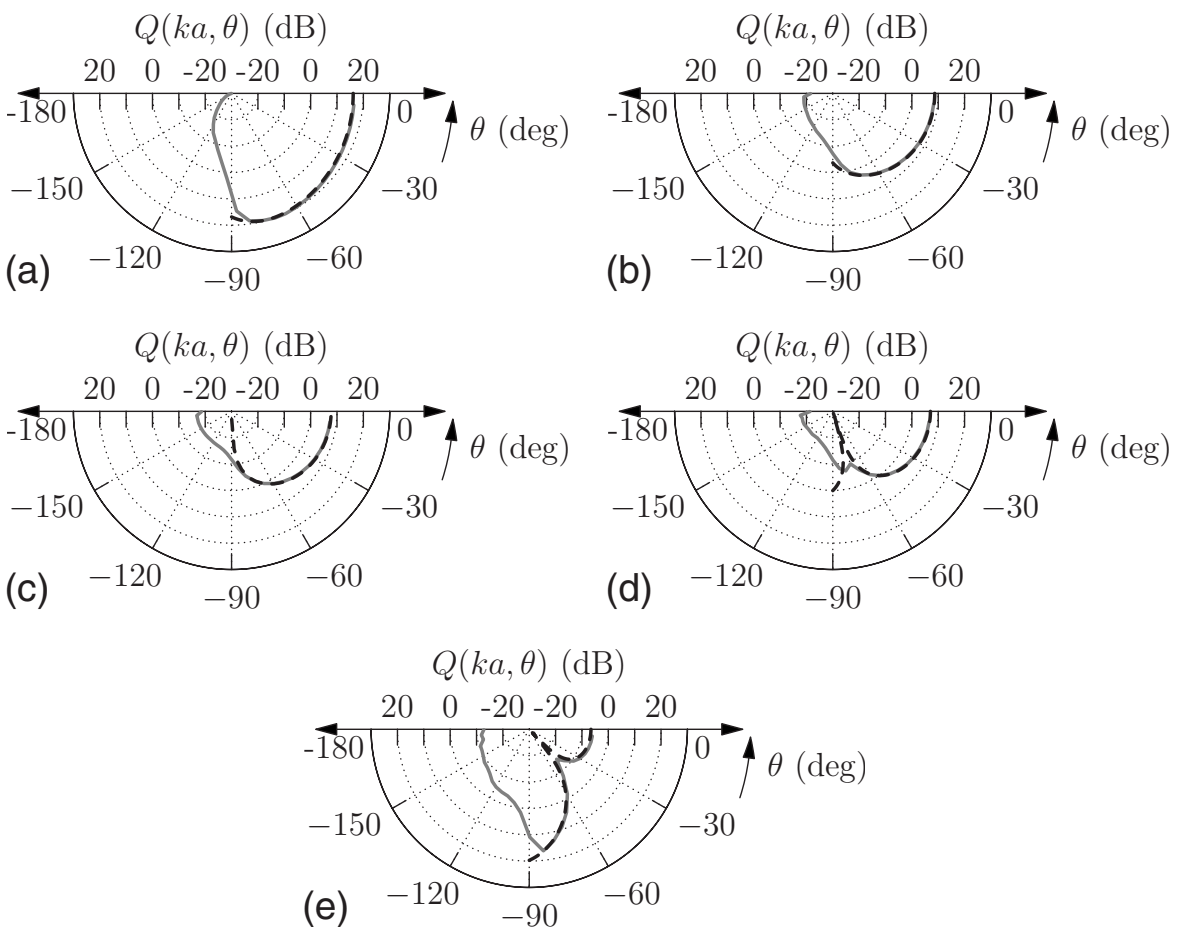

FIG. 6. [(a)-(e)] Multimode directivity function $Q(k a, \theta)$ for sound radiated from a circular duct with uniform flow. The source is a uniform distribution of incoherent axial dipoles. Results are shown for $k a=50$ and for five different values of Mach number $M$. The solid line represents the exact numerical solution given by Eq. (11) (see Sec. III). The dashed line represents the high-frequency asymptotic solution given by Eq. (31b). 

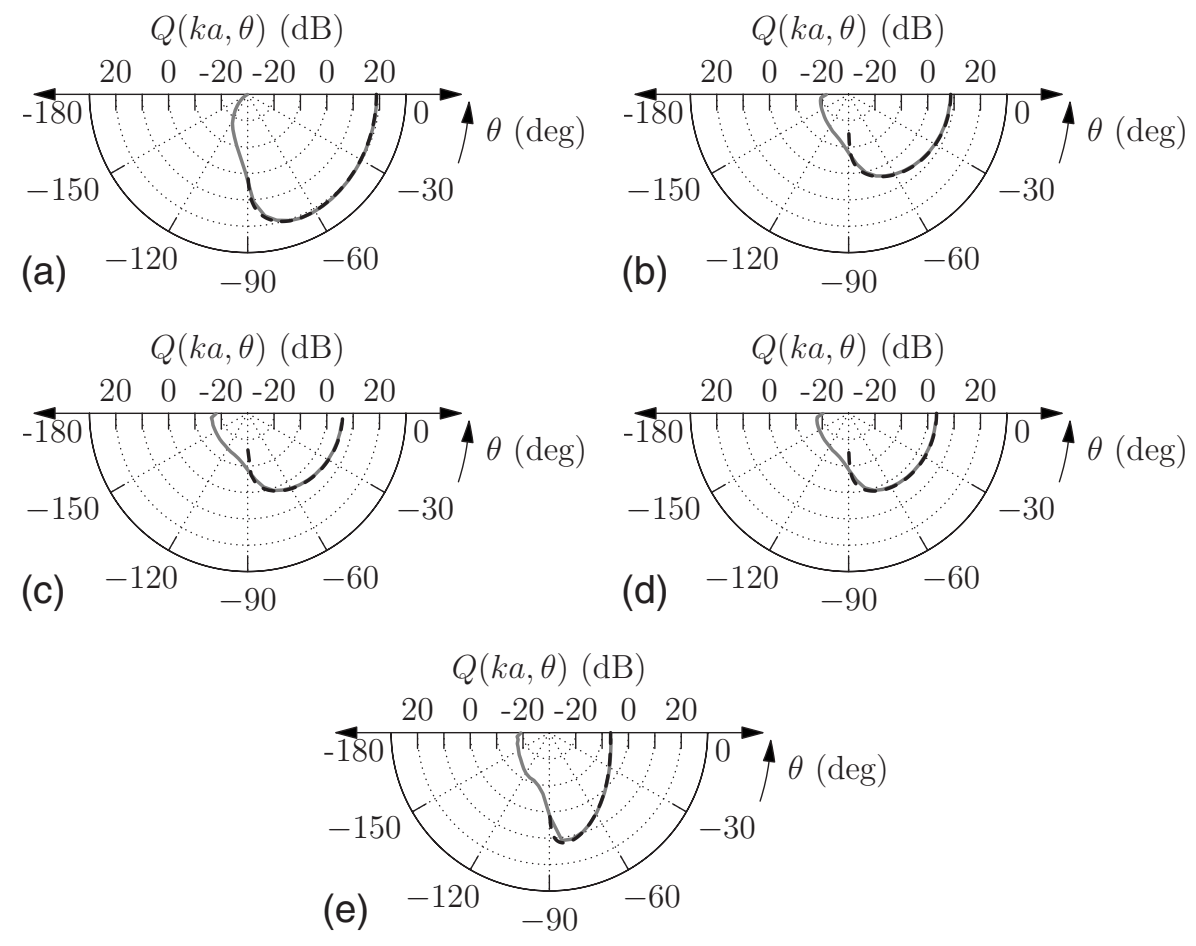

FIG. 7. [(a)-(e)] Multimode directivity function $Q(k a, \theta)$ for sound radiated from a circular duct with uniform flow. The source is equal power per mode. Results are shown for $k a=50$ and for five different values of Mach number $M$. The solid line represents the exact numerical solution given by Eq. (11) (see Sec. III). The dashed line represents the high-frequency asymptotic solution given by Eq. (31c).

From Eq. (10b) for the mode amplitude distribution due to axial dipoles, this angle corresponds to the modal propagation angle $\theta_{0}$ at which $E\left\{\left|A_{m n}\left(\alpha\left(\theta_{0}\right)\right)\right|^{2}\right\}=0$. This may be shown to be at the angle at which the modal group velocity is zero.

Finally, for equal power per mode, the behavior is similar to that for a uniform distribution of incoherent monopoles (Fig. 7).

\section{CONCLUSIONS}

In this paper, the directivity function of multimode sound radiation from an unflanged, semi-infinite circular duct with uniform flow is investigated. Both inlet $(M<0)$ and exhaust $(M>0)$ cases are examined. This problem is of importance in the understanding of, for example, the radiation of fan broadband noise from aero-engine ducts. The behavior of the multimode radiation is considered for a general family of mode amplitude distribution function. Special cases are incoherent monopoles or axial dipoles uniformly distributed over the duct cross section, and the case of equal power per mode. A non-dimensional directivity function $Q(k a, \theta)$ is defined, normalized with respect to the total radiated acoustic power. Analytic expressions for $Q$ are derived for these three source models that are valid in the highfrequency limit and in the forward arc. The dependence on the radiation polar angle $\theta$ and flow Mach number is revealed explicitly. The presence of a uniform mean flow in a duct has been demonstrated, through the mechanism of convective amplification, to alter the total radiated sound power compared with when flow is absent. The paper has shown that the variation in sound power radiation with $M$ is identical for the mode distributions of "equal energy per mode" and a distribution of incoherent monopoles. Increases of less than $3 \mathrm{~dB}$ are predicted for flow speeds of $M<0.5$ for these two source models. However, an incoherent distribution of axial dipole sources has been shown to radiate less sound power for exhaust ducts for flow speed $M<0.5$ but radiate more sound power at higher flow speeds compared with when flow is absent. The effect of flow has been found to produce significant distortions of the far-field directivity patterns. Of the three mode distributions models investigated in detail in this paper the directivity patterns due to axial dipole sources are predicted to be most sensitive to flow. In the exhaust duct, the presence of flow has been shown to introduce an additional null in the directivity pattern corresponding to the main radiation lobe of the modes of zero group velocity. In general, however, the paper has shown that effects of flow may be neglected for flow Mach number of less than $100 \mathrm{~m} \mathrm{~s}^{-1}(M<0.3)$.

\section{ACKNOWLEDGMENTS}

The authors wish to thank Professor R. J. Astley (ISVR), and one of the referees, for their guidance regarding the use of the Lorentz transform to derive Eqs. (7) and (8).

\section{APPENDIX: DERIVATION OF $\delta N(\theta)$}

In this appendix an expression for the number of modes in a rigid-walled circular duct with propagation angles between $\theta$ and $\theta+\delta \theta$, in the high-frequency limit $k a \rightarrow \infty$, is derived. The duct is assumed to contain a uniform mean flow with Mach number $M$. The derivation makes use of previous work by Rice ${ }^{15}$ on the total number of cut-on modes with 
flow. Rice's result is expressed in terms of the cut-off ratio $\xi$, which is related to the cut-on ratio $\alpha$ of Eq. (3) by

$$
\alpha=\sqrt{1-\xi^{-2}},
$$

and is in the range $\xi \geq 1$ for the propagating modes. Note that in this appendix we follow Rice's nomenclature, so the analysis is in terms of cut-off ratio $\xi$. Following Rice, ${ }^{15}$ the total number of cut-on modes $N(\xi)$ having a cut-off ratio below a particular value $\xi$ is given by

$$
N(\xi)=\frac{\left(\frac{1}{2} k a\right)^{2}}{\xi^{2}} .
$$

Note that $\left(\frac{1}{2} k a / \beta\right)^{2}$ is the high- $k a$ asymptotic expression for the total number of propagating modes in the duct.

Differentiating Eq. (A2) yields the following equation for the number of modes $\delta N(\xi)$ with cut-off ratio between $\xi$ and $\xi+\delta \xi$, where $\delta \xi$ is small:

$$
\delta N(\xi)=\frac{d N(\xi)}{d \xi} \delta \xi=-2 \frac{\left(\frac{1}{2} k a\right)^{2}}{\xi^{3}} \delta \xi .
$$

The cut-off ratio may be related to the modal propagation angle using Eq. (19) first derived by Rice et al., ${ }^{14}$

$$
\cos \theta=\frac{\alpha \beta}{\sqrt{1-M^{2} \alpha^{2}}}
$$

The number of modes $\delta N(\theta)$ with propagation angles between $\theta$ and $\theta+\delta \theta$ may therefore be obtained by substituting Eqs. (A4) and (A1) into Eq. (A3) and noting that $\delta \xi$ $=(d \xi / d \alpha)(d \alpha / d \theta) \delta \theta$, to give

$$
\delta N(\theta)=\frac{\left(\frac{1}{2} k a\right)^{2} \sin 2 \theta}{\left(1-M^{2} \sin ^{2} \theta\right)^{2}} \delta \theta .
$$

${ }^{1}$ P. Joseph and C. L. Morfey, "Multimodal radiation from an unflanged, semi-infinite circular duct," J. Acoust. Soc. Am. 105, 2590-2600 (1999).

${ }^{2}$ G. F. Homicz and J. A. Lordi, "A note on the radiative directivity patterns of duct acoustic modes," J. Sound Vib. 41, 283-290 (1975).

${ }^{3}$ E. J. Rice, "Multimodal far-field acoustic radiation pattern," AIAA J. 16, 906-911 (1978).

${ }^{4}$ R. M. Munt, "The interaction of sound with a subsonic jet issuing from a semi-infinite cylindrical pipe," J. Sound Vib. 83, 609-640 (1977).

${ }^{5}$ R. M. Munt, "Acoustic transmission properties of a jet pipe with subsonic jet flow: I. The cold jet reflection coefficient," J. Sound Vib. 142, 413-436 (1990).

${ }^{6}$ S. W. Rienstra, "Sound radiated from annular duct in flow," J. Sound Vib. 94, 267-288 (1984).

${ }^{7}$ G. Gabard and R. J. Astley, "Theoretical model for sound radiation from annular jet pipes: Far- and near-field solutions," J. Fluid Mech. 549, 315341 (2006).

${ }^{8}$ D. W. Bechert, "Sound-absorption caused by vorticity shedding, demonstrated with a jet flow," J. Sound Vib. 70, 389-405 (1980).

${ }^{9}$ Rice's cut-off ratio $\xi$ is uniquely related to our cut-on ratio $\alpha$; see the Appendix, Eq. (A1).

${ }^{10}$ J. M. Tyler and T. G. Sofrin, "Axial flow compressor noise studies," SAE Trans. 70, 309-332 (1962).

${ }^{11}$ C. J. Chapman, "Similarity variables for sound radiation in a uniform flow," J. Sound Vib. 233, 157-164 (2000).

${ }^{12}$ P. Joseph, C. L. Morfey, and C. R. Lowis, "Multi-mode sound transmission in ducts with flow," J. Sound Vib. 264, 523-544 (2003).

${ }^{13}$ C. L. Morfey, "Sound transmission and generation in ducts with flow," J. Sound Vib. 14, 37-55 (1971).

${ }^{14}$ E. J. Rice, M. F. Heidmann, and T. G. Sofrin, "Modal propagation angles in a cylindrical duct with flow and their relation to sound radiation," presented at the 17th Aerospace Sciences Meeting, New Orleans, LA, 15-17 January (1979), AIAA Paper No. 79-0183.

${ }^{15} \mathrm{E}$. J. Rice, "Modal density function and number of propagating modes in ducts," J. Acoust. Soc. Am. 60, S112 (1976). 